\title{
Article \\ EXACT SOLUTIONS FOR THE AXIAL PRESSURE DROP IN ISOTHERMAL CHANNELS WITH MAXWELL SLIP FLOW
}

\author{
Alex C. Hoffmann ${ }^{1, t}+$ (DD000-0002-4629-8047 and Stamatina Karakitsiou ${ }^{2, *}$ and Bodil Holst ${ }^{3}$ \\ 1 Dept. of Physics and Technology, University of Bergen, Bergen, Norway \\ * Correspondence: alex.hoffmann@uib.no; Tel.: +47-90832490 \\ $\ddagger$ These authors contributed equally to this work.
}

\begin{abstract}
Expressions for the axial pressure profiles in a cylindrical channel and between parallel plates or a rectangular channel with large aspect ratio, with Maxwell slip gas flow are derived from first $p$ rinciples. The resulting expressions, which only involve the inlet and outlet pressures and the channel dimensions, will be useful in modelling or simulations of channel flows at Knudsen numbers in the range $10^{-3}-0.1$, such as in MEMS and NEMS. The expression for a cylindrical channel is validated by deriving from it an expression for the channel mass flow, which is shown to agree with a known expression for the mass flow through cylindrical channels with Maxwell slip flow. The expression for flow between parallel plates is found to agree with the zeroth order relation derived by Arkilic et al. using perturbation analysis. The effect of the accommodation coefficient on the pressure profile in a cylindrical channel is shown.
\end{abstract}

Keywords: Maxwell slip flow; microchannels; pressure profile

\section{Introduction}

An upsurge in the development of micro-and nanoelectromechanical devices (MEMS and NEMS) has kindled renewed interest in the flow of fluids at moderately high Knudsen numbers through channels and crevices.

In many modern design applications, such as in the design of MEMS and NEMS [1-3], it is necessary to characterize and model the flow of fluids in narrow channels. For this it is useful to know the pressure profile in channel flow at Knudsen numbers whereby the flow is in the Maxwell slip regime, since this is often the regime into which the flows in such devices fall in practise.

Expressions for the pressure profiles for Maxwell slip flow in channels, even of simple geometries, have not yet been published.

Arkilic et al. [4] in a highly cited paper derived an expression for the axial pressure profile in slip flow, based on a 2-D model and perturbation analysis in a rectangular channel of very large aspect ratio, it is stated in that paper that their expression is accurate to zeroth orderr. Gallis and Torczynsky [5] derived an expression for the pressure profile in the slip flow regime based on a more advanced slip expression than the one of Maxwell, involving four dimensionless parameters, determined so that the mass flow in the channel would match benchmark values in the different high-Knudsen-number flow regimes. Their expression is implicit in the pressure, the authors state it may be inverted, but that the "the resulting expression is too complicated to be useful".

In this present paper, explicit expressions for the axial pressure profiles in a cylindrical channel and a rectangular channel with large aspect ratio, both of uniform temperature, with Maxwell slip flow will be derived from first principles. The accuracy of the derived expressions is only limited by the accuracy of the Maxwell slip assumption itself. 
34

35

\subsection{Flow in a cylindrical channel}

A standard momentum balance on an element differential in the axial direction to analyze laminar pipe flow, with a no-slip wall boundary condition, results in [6]:

$$
\frac{d p}{d z}=-\frac{2}{r}\left(-\mu \frac{d u}{d r}\right)
$$

giving by integration:

$$
\int_{0}^{u} d u^{\prime}=\frac{d p}{d z} \frac{1}{2 \mu} \int_{R}^{r} r^{\prime} d r^{\prime}
$$

The wall slip for Maxwell slip is $([7,8]): u_{w}=-\left.\zeta \frac{d u}{d r}\right|_{r=R}$ with the factor $\zeta$ expressed as: $\zeta=\frac{\zeta_{0}}{p}$ and the factor $\left.\frac{d u}{d r}\right|_{r=R}$ evaluated from the no-slip solution. Inserting this expression for $u_{w}$ in the lower integration limit on the RHS of (2)gives:

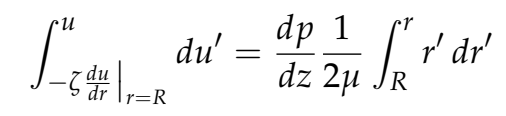

giving:

$$
u+\left.\zeta \frac{d u}{d r}\right|_{r=R}=-\frac{d p}{d z} \frac{1}{4 \mu}\left(R^{2}-r^{2}\right)
$$

From the no-slip DE, (1):

$$
\frac{d u}{d r}=\left.\frac{r}{2 \mu} \frac{d p}{d z} \Rightarrow \frac{d u}{d r}\right|_{r=R}=\frac{R}{2 \mu} \frac{d p}{d z}
$$

Ref. [9], together with others, give $\zeta=\frac{2-\sigma_{V}}{\sigma_{V}} \lambda . \lambda$ is the mean free path in the gas and $\sigma_{V}$ is the momentum accommodation coefficient (see [10] and also below), which in general depends on the nature of the gas, the channel wall material and the Knudsen number. In many cases $\sigma_{V} \approx 0.8([10,11])$. Filling in, this makes the solution for the gas velocity, $u$ :

$$
u=-\frac{d p}{d z} \frac{1}{4 \mu}\left(R^{2}-r^{2}\right)-\frac{2-\sigma_{V}}{\sigma_{V}} \lambda \frac{R}{2 \mu} \frac{d p}{d z} .
$$

The mass flow, $\dot{m}_{t}=\int_{0}^{R} \rho u 2 \pi r d r$, becomes:

$$
\dot{m}_{t}=-\frac{\pi \rho R^{3}}{8 \mu \sigma_{V}}\left(4 \lambda\left(2-\sigma_{V}\right)+\sigma_{V} R\right) \frac{d p}{d z} .
$$

$\rho=\frac{p M}{\mathbb{R} T}=\frac{p m}{k_{B} T}$ where $M$ is the molar mass, $m$ is the molecular mass, $\mathbb{R}$ is the universal gas constant, $k_{B}$ is the Boltzmann constant and $\lambda=\frac{k_{B} T}{\sqrt{2} \pi d^{2} p}$ ([8]), with $d$ the molecular collisional diameter, giving:

$$
\dot{m}_{t}=-\frac{\pi p m R^{3}}{k_{B} T 8 \mu \sigma_{V}}\left(4 \frac{k_{B} T}{\sqrt{2} \pi d^{2} p}\left(2-\sigma_{V}\right)+\sigma_{V} R\right) \frac{d p}{d z}
$$


which can be rewritten to:

$$
\dot{m}_{t}=A \frac{d p}{d z}+B p \frac{d p}{d z}
$$

where, after simplification,

$$
A=-\frac{m R^{3}\left(2-\sigma_{V}\right)}{\sqrt{8} d^{2} \mu \sigma_{V}} \quad \text { and } \quad B=-\frac{\pi m R^{4}}{8 \mu k_{B} T} .
$$
linear.

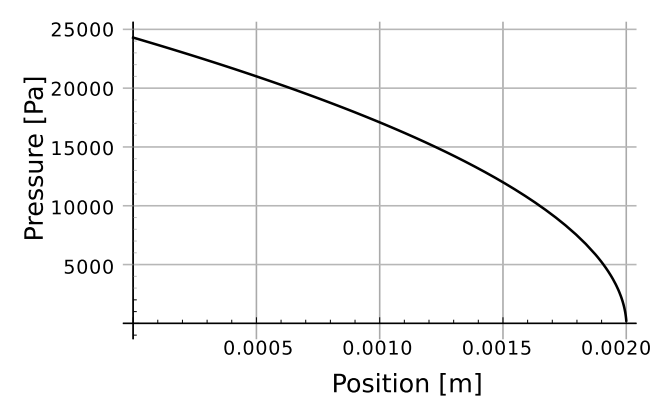

There are two solutions to this equation, we take the positive one, since this is the one satisfying the boundary conditions at the two ends of the channel, giving:

$$
p=-\frac{A+\sqrt{\left(A+B p_{0}\right)^{2}-\frac{B\left(p_{0}-p_{L}\right)\left(2 A+B\left(p_{0}+p_{L}\right)\right) z}{L}}}{B}
$$

resulting in $p=p_{0}$ for $z=0$ and $p=p_{L}$ for $z=L$ agreeing with the boundary conditions (remember the values of $A$ and $B$ are negative). A plot of pressure along the channel, see Fig. 1, shows a similar shape to that for Poiseuille flow for flow where the inlet Knudsen number is relatively low $(0.001$, the lower limit of the range where Maxwell slip is normally applied), while it becomes more linear for flow at higher Knudsen numbers as expected, since in the molecular-flow limit the pressure profile is

Figure 1. Pressure profiles for slip flow of argon in a cylindrical channel of diameter $325 \mu \mathrm{m}$ and length $2 \mathrm{~mm}$. Left: inlet Knudsen number: $1.0 \times 10^{-3}$ Right: inlet Knudsen number: 0.0244 . In both cases the outlet Knudsen number is 0.1

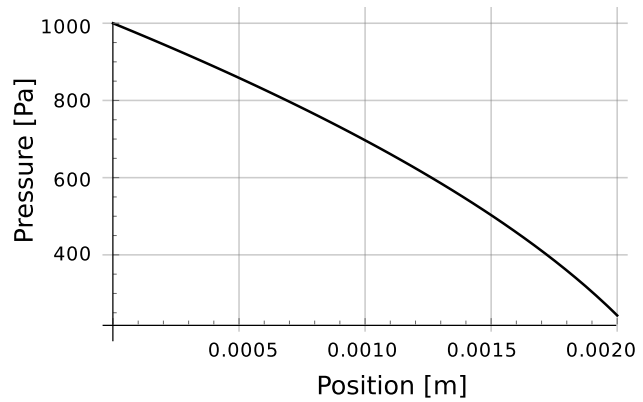

\subsection{Flow between parallel plates or in a wide, rectangular channel}

Slip flow in a wide rectangular channel or between parallel plates has been the subject of a number of investigations (e.g. [4,12-14]) and is relevant in a variety of MEMS and NEMS applications. The derivation in this section of the stream-wise pressure profile will follow the general method of the 
55

56

derivation in the previous section for flow in a cylindrical channel, starting with Poiseuille flow, since the result of this is used also here for quantifying the slip term.

The flow will be assumed two-dimensional, thus the effect of the side walls (left and right walls in the front view in Fig. 2) on the flow is neglected.

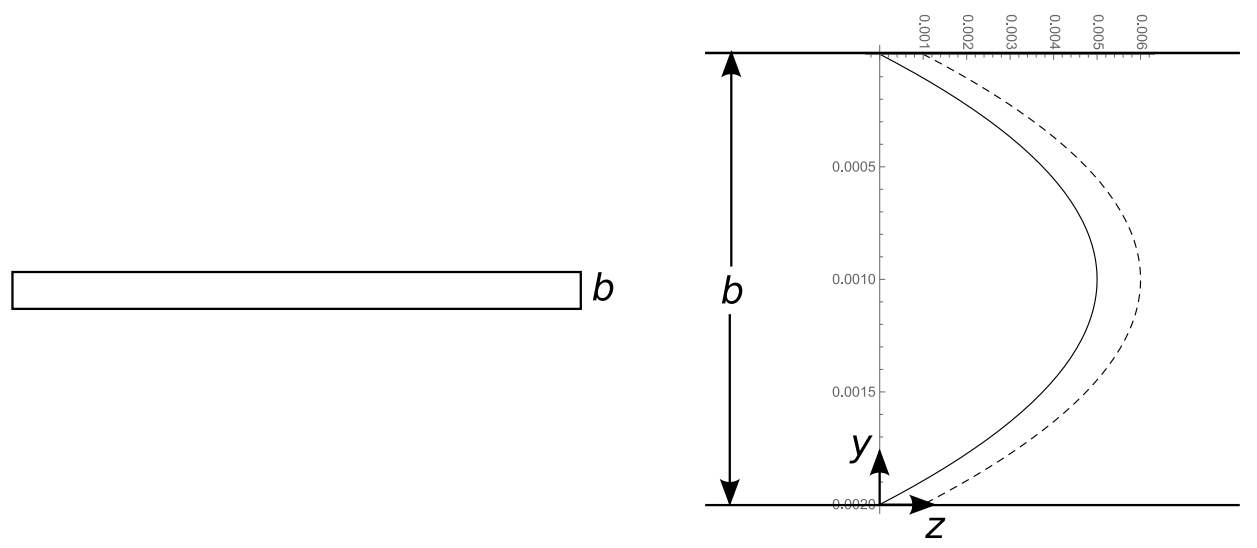

Figure 2. Schematic of a wide rectangular channel. Left: front view. Right: expanded side view showing velocity profiles for Poiseuille flow (fully drawn line) and Maxwell slip flow (broken line)

The standard momentum balance, which reduces to a force balance, since the convective momentum flows in and out are equal, on an element, differential in both the $z$-direction (in anticipation that the pressure gradient varies in this direction) and the $y$-direction, for Poiseuille flow gives the differential equation for the fluid velocity:

$$
\frac{d p}{d z}=-\mu \frac{d^{2} u}{d y^{2}}
$$

Integrating twice in the y-direction, with the no-slip boundary conditions $u=\left.0\right|_{y=0 \text { or } y=b}$ gives:

$$
u=\frac{1}{2 \mu} \frac{d p}{d z}\left(b y-y^{2}\right)
$$

giving a velocity gradient magnitude at the walls of

$$
\left|\frac{d u}{d y}\right|_{y=0 \text { or } y=b}=\frac{b}{2 \mu} \frac{d p}{d z}
$$

Solving Eq. (11) with the boundary condition at the walls: $u_{w}=\zeta\left|\frac{d u}{d r}\right|_{y=0 \text { or } y=b}$ gives:

$$
u=\frac{1}{2 \mu} \frac{d p}{d z}\left(b y-y^{2}\right)+\zeta\left|\frac{d u}{d r}\right|_{y=0 \text { or } y=b},
$$

which just adds the slip velocity to the Poiseuille velocity profile. This profile is shown in Fig. 2. Filling in for $\left|\frac{d u}{d r}\right|_{y=0 \text { or } y=b}$ from Eq.(13) and that $\zeta=\frac{2-\sigma_{V}}{\sigma_{V}} \lambda$ (see the previous section) the mass flow per unit width, $\dot{m}_{p}=\int_{0}^{b} \rho u d y$ becomes:

$$
\dot{m}_{p}=\frac{b^{3} \rho}{12 \mu} \frac{d p}{d z}-\frac{b^{2} k_{B} T \rho}{2 \sqrt{2} d^{2} \pi \mu p} \frac{d p}{d z}+\frac{b^{2} k_{B} T \rho}{\sqrt{2} d^{2} \pi \mu \sigma_{V} p} \frac{d p}{d z}
$$


using $\rho=\frac{p M}{\mathbb{R} T}=\frac{p m}{k_{B} T}, \dot{m}_{p}$ can be written in the same form as $\dot{m}_{t}$ above (Eq. 7), namely:

$$
\dot{m}_{p}=A \frac{d p}{d z}+B p \frac{d p}{d z}
$$

this time with

$$
A=\frac{m b^{2}\left(\frac{1}{\sigma_{V}}-\frac{1}{2}\right)}{\sqrt{2} d^{2} \pi \mu} \quad \text { and } \quad B=\frac{b^{3} m}{12 k_{B} T \mu} .
$$

The derivation of a second-order differential equation for $p$ then follows the derivation in the previous section leading to Eq. (9). In this case, however, the two constants A and B are both positive, so that the other of the two solutions of Eq. (9) is the physically relevant one satisfying the end conditions, namely:

$$
p=\frac{-A+\sqrt{\left(A+B p_{0}\right)^{2}-\frac{B\left(p_{0}-p_{L}\right)\left(2 A+B\left(p_{0}+p_{L}\right)\right) z}{L}}}{B}
$$

For Maxwell slip flow in a wide rectangular channel, or between parallel plates, the pressure profile is thus given by Eq. (18) with the expressions for $A$ and $B$ given in Eq. (17).

\section{Discussion}

\subsection{Cylindrical-channel flow}

Rather than trying to compare with experiment, which can be somewhat ambiguous, we can show that the expression for cylindrical channel flow is correct by comparing an expression for the mass flow derived from Eq. (10) with an analytical expression for the mass flow through cylindrical channels with slip flow, which is known although an expression for the pressure profile was not known till now.

Substituting the pressure profile given in Eq. (10) into Eq. (7), and finding the mass flow based on the channel inlet conditions by setting $z=0$ and using $\left.\frac{d p}{d z}\right|_{z=0}$ and $p=p_{0}$, gives for the mass flow, after simplification:

$$
\dot{m}=-\frac{\left(p_{0}-p_{L}\right)\left(B p_{L}+B p_{0}+2 A\right)}{2 L}
$$

which is positive, since $A$ and $B$ are negative. This result can be found using the axial pressure gradient anywhere along the channel, not only at the inlet. The analysis in [8] results in an expression for the mass flow through a channel with slip flow:

$$
\dot{m}=\frac{\pi\left(p_{0}-p_{L}\right) R^{4}}{8 \mu L}\left(\frac{\left(p_{0}+p_{L}\right) M}{2 \mathbb{R} T}\right)\left(1+\frac{8 \zeta_{0}}{R\left(p_{0}+p_{L}\right)}\right) .
$$

Substituting into Eq. (19) the expressions for $A$ and $B$ given in Eq. (8) and substituting into Eq. (20) that $\zeta_{0}=\frac{\left(2-\sigma_{V}\right)}{\sigma_{V}} p \lambda$, with $\lambda$ given in Sect. 2.1, shows, after some rearrangement, that the two expressions for the mass flow are identical showing the correctness of the derived expression for the axial pressure profile.

\subsection{Flow between parallel plates}

Since the expression derived in this paper for the pressure profile in the flow direction is exact, it can be used to check the accuracy of the solution of [13]. However the expression in [13] is complex to work out, since it requires the outlet velocity of the gas to calculate the outlet Reynolds number and the Mach number to work out the outlet Knudsen number, $K$ (see below), which is required in their expression for the profile in the flow direction of the dimensionless pressure, $\tilde{p}_{0} \equiv \frac{p}{p_{L}}$ :

$$
\tilde{p}_{0}(\tilde{x})=-6 \sigma K+\sqrt{(6 \sigma K)^{2}+(1+12 \sigma K) \tilde{x}+\left(\mathcal{P}^{2}+12 \sigma K \mathcal{P}\right)(1-\tilde{x})}
$$


where the subscript 0 on the left-hand-side does not signify the pressure at the channel inlet, as it does in this paper, but rather that the solution is accurate to zeroth order as stated by Arkilic et al. $\tilde{x} \equiv \frac{z}{L}, \mathcal{P} \equiv \frac{p_{0}}{p_{L}}, \sigma \equiv \frac{2-\sigma_{V}}{\sigma_{V}}, K$ is the outlet Knudsen number based on the channel height, given in [13] as $K=\sqrt{\frac{\pi \gamma}{2}} \frac{M}{R}$, with $M$ and $R$ the outlet Mach number and Reynolds number, respectively, and $\gamma$ is the adiabatic heat capacity ratio.

Making the substitutions indicated into Eq. (21) but using the expression for the Knudsen number: $K=\frac{\lambda}{b}$ with the mean free path in the gas as: $\lambda=\frac{k_{B} T}{\sqrt{2} \pi d^{2} p}$, rather than the expression for $K$ used in [13], and substituting into Eq. (18) from Eq. (17) it is possible to show that the expressions derived in this work and that given in [13] for the axial pressure profile are identical. The advantage of the derivation presented here is that it is more direct and transparent, the only assumption involved being that of Maxwell slip flow, and furthermore that the derivation in this paper is applicable to other types of conduits, as shown for the case of cylindrical conduits above. The pressure profiles resulting from the expression derived here, together with a graphical confirmation that the expression of Arkilic et al and that in this paper give the same results is given in Fig. 3
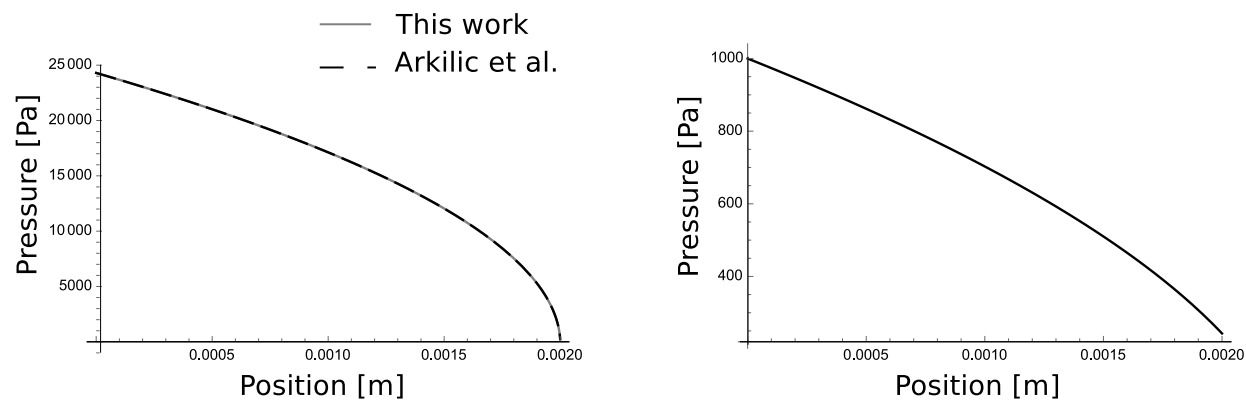

Figure 3. Pressure profiles for slip flow between two parallel plates $325 \mu \mathrm{m}$ apart according to Eq(18) with (17). Left the profile is plotted together with that of Arkilic et al., confirming graphically that their expressions is the same as the one derived here.

(6) paral the ther

$$
\text { p }
$$

2 4. Effect of the accommodation coefficient on the axial pressure profile

In much of the literature the accommodation coefficient is taken as 1.0. Intuitively one would expect a lower flow and, perhaps, a more non-linear axial pressure profile for the same pressure drop for a higher accommodation coefficient. However, it turns out that the effect changing $\sigma_{V}$ from $0.8 \mathrm{t} 0$ 1.0 has very little effect on the actual shape of the profile and this only reduces the the mass flow, for like pressure drop over the channel, by $0.85 \%$. To see a significant effect on the shape of the profile, $\sigma_{V}$ had to be reduced substantially. In Fig. 4 the effect of reducing $\sigma_{V}$ to 0.1 is shown. The effect is to make the profile more linear, as one might intuitively expect. The mass flow through the channel by like pressure drop increased by $23 \%$ making this change. We conclude that the practice of taking the accommodation coefficient as unity does not introduce much error.

\section{Conclusions}

- A solution method for the axial pressure profiles in conduits with Maxwell slip flow is presented and applied to the two types of conduits with the most practical applicability: a conduit with 


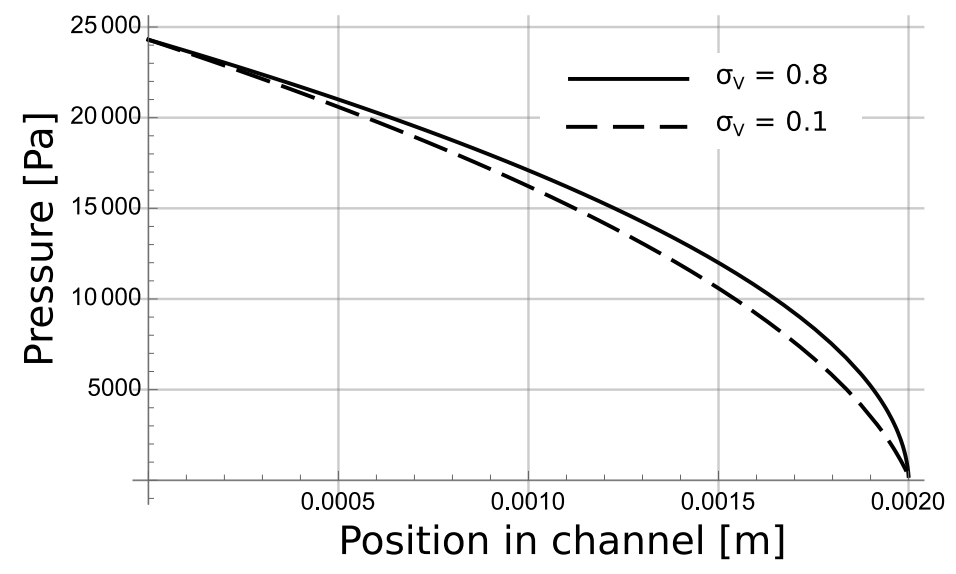

Figure 4. Pressure profiles for slip flow in a cylindrical channel with diameter $325 \mu \mathrm{m}$, effect of the accommodation coefficient.

cylindrical cross-section and flow between two parallel plates, which can also be used for flow in a rectangular conduit with large aspect ratio. The solutions have been verified by deriving from them expressions that could be compared with literature.

- The solutions are useful for the design of MEMS and NEMS, and can be used to pinpoint the positions of regime transitions in conduits with flow with high pressure ratios between inlet and outlet.

- the pressure profiles in cylindrical channels and rectangular channels with large aspect ratio under the Maxwell slip assumption, for like characteristic dimensions (tube diameter and channel height, respectively), have been found to be very similar but not identical.

- The accommodation coefficient has been found to have only a minor effect on the pressure profile in Maxwell slip flow. The accommodation coefficient had to be reduced substantially to see a significant effect.

\section{References}

1. Ho, C.M.; Tai, Y.C. Micro-electro-mechanical-systems (MEMS) and fluid flows. Annual Review of Fluid Mechanics 1998, 30, 579-612.

2. Monty, C.N.; Ilwhan, O.I.; Masel, R.I. Optimization of a Multiphase Sensor for Detection of Phosphonates in Air. AICheE Journal 2010, 56, 241-247.

3. Ebrahimi, A.; Roohi, E. DSMC investigation of rarefied gas flow through diverging micro- and nanochannels. Microfluidics and Nanofluidics 2017, 21, 21:18.

4. Arkilic, E.B.; Schmidt, M.A.; Breuer, K.S. Gaseous slip flow in long microchannels. Journal of Microelectromechanical Systems 1997, 6, 167-178.

5. Gallis, M.A.; Torczynsky, J.R. DSMC-based expression for the gas mass flow rate and pressure profile in a microscale tube. Physics of Fluids 2012, 24, 012005-1-012005-21.

6. McCabe, W.L.; Smith, J.; Hariott, P. Unit operations of chemical engineering, 7 ed.; McGraw-Hill: London, 2004.

7. Pollard, W.G.; Present, R.D. On gaseous sef-diffusion in long capillary tubes. Physical Review 1948, 73, 762-774.

8. Bird, R.B.; Stewart, W.E.; Lightfoot, E.N. Transport phenomena, 2 ed.; John Wiley \& Sons: New York, 2002.

9. Roy, S.; Raju, R.; Chuang, H.F.; Cruden, B.A.; Meyyappan, M. Modeling gas flow through microchannels and nanopores. Journal of Applied Physics 2003, 93, 4870-4879.

10. Karniadakis, G.; Beskok, A.; Aluru, N. Microflows and Nanoflows. Fundamentals and Simulation; Springer Verlag: New York, 2005.

11. Roth, A. Vacuum technology; Elsevier Science B.V: Amsterdam, 1990.

12. Harley, J.; Bau, H.H.; Zemel, J.N. Gas-flow in micro-channels. Journal of Fluid Mechanics 1995, 284, 257-274. 
138 13. Arkilic, E.B.; Schmidt, M.A.; Breuer, K.S. Mass flow and tangential momentum accommodation in silicon 139 micromachined channels. Journal of Fluid Mechanics 2001, 437, 29-43.

140 14. Shi, T.; Wang, L.; Guo, Z.L. Lattice Boltzmann modeling of microscale oscillating Couette flow. Acta Physica $141 \quad$ Sinica 2014, 63, Art. 214703. 